\title{
Risk factors and prediction model construction of post-operative atrial fibrillation after thoracic surgery in Chinese patients
}

\author{
Yunling Lin ${ }^{1}$, Jianmin Sun², Xun Yuan¹, Hui Liü
} \author{
${ }^{2} F u j i a n$ Medical University, FuZhou Fujian, China \\ Submitted: 19 May 2020 \\ Accepted: 1 December 2020 \\ Arch Med Sci \\ DOI: https://doi.org/10.5114/aoms/131087 \\ Copyright $\odot 2020$ Termedia \& Banach
}

${ }^{1}$ Department of Cardiology, Fujian Medical University Union Hospital, Fujian Institute of Coronary Artery Disease, Fujian Heart Medical Center, Fuzhou, Fujian, China

${ }^{3}$ Fujian Medical University Union Hospital, Fuzhou, Fujian, China

\section{Abstract}

Introduction: The purpose of this study was to analyze the risk factors of post-operative atrial fibrillation (POAF) after thoracic surgery, and to build a predictive model for accurate preoperative identification of high-risk patients.

Material and methods: In this study, data of 2072 patients with pulmonary masses and esophageal cancer who attended our hospital in the period from January 1, 2017 to December 31, 2018 were analyzed retrospectively. According to whether AF occurred after the operation, the patients were divided into atrial fibrillation (AF) and non-AF (NAF) groups. The general information (age, sex, height, etc.), previous medical history (chronic lung disease, hypertension, etc.), medication history, preoperative ultrasound and cardiogram results, and preoperative and postoperative electrocardiogram (ECG) were collected. The operation mode, resection scope, histopathology and hospitalization were recorded. Univariate and multivariate logistic regression were used to screen out the risk factors of AF and establish a prediction model.

Results: The incidence of POAF was $5.98 \%$. Univariate analysis showed that sex, age, body mass index, left atrial diameter and operation organ were the risk factors of POAF. The above factors were included in the multivariate logistic regression analysis, and the results showed that male sex, age, anteroposterior diameter of left atrium and surgical organs were related to POAF. On this basis, a POAF prediction model was constructed, which had good discrimination and calibration. The area under the curve (AUC) is 0.784 with $95 \% \mathrm{Cl}: 0.746-0.822$.

Conclusions: The prediction model of POAF based on the risk factors selected in this study can accurately predict the occurrence of AF after thoracic surgery.

Key words: atrial fibrillation, thoracic surgery, risk factors, predictive model.

\section{Introduction}

Postoperative atrial fibrillation (POAF) is a common complication after thoracic surgery with the incidence of about 12-44\% [1]. POAF may cause hemodynamic disturbances and heart failure in the acute phase,

\author{
Corresponding author: \\ Yunling Lin PhD \\ Department of Cardiology \\ Fujian Medical \\ University Union Hospital \\ Fujian Institute of \\ Coronary Artery Disease \\ Fujian Heart \\ Medical Center \\ Fuzhou, Fujian \\ China, 350001 \\ E-mail: lyhdsia116@126.com
}


and is related to prolonged hospital stay, increased medical expenses and increased mortality [2-4]. Studies have shown that in future, patients with POAF after thoracic surgery have an 8-fold greater risk of developing $A F$ than those without POAF [5]. POAF has a similar risk of thromboembolism compared to non-valvular AF [6], and the risk of long-term stroke is significantly increased [2]. Although preventive drugs ( $\beta$-blockers, diltiazem, amiodarone, etc.) for POAF have been used in the clinic, their intervention effect is still controversial [7-9]. Screening the preoperative risk factors of POAF is conducive to the early risk assessment and stratification of POAF, so as to carry out preventive intervention for high-risk groups, reduce the occurrence of POAF, promote the postoperative rehabilitation of patients, and reduce the incidence of thrombosis and other adverse events [8]. The POAF score is a tool for predicting the risk of postoperative atrial fibrillation after cardiac surgery [10]. At present, there are few prediction tools for AF after thoracic non-cardiac surgery, and most of them are based on European and American populations. The incidence of POAF is related to race. Nowadays there are few studies on the occurrence and risk factors of POAF in Asian population with a small sample size. In this study, the risk factors of POAF were investigated by retrospective analysis of the relevant data of patients with pulmonary masses and esophageal cancer who underwent thoracic surgery in our hospital, so as to lay a foundation for the establishment of the prediction model of POAF.

\section{Material and methods}

\section{Patients}

This study was approved by the Ethics Committee of the Fujian Medical University Union Hospital (2016020). All participants provided informed consent. A total of 1834 patients with pulmonary masses and esophageal cancer who attended our hospital in the period from January 1, 2018 to December 31, 2018 were enrolled in our retrospective study. Inclusion criteria: (1) patients undergoing thoracic surgery in our hospital, with lung cancer confirmed by preoperative diagnosis pathology; (2) patients with esophageal cancer confirmed by preoperative diagnosis; (3) patients with lung cancer who underwent thoracotomy or thoracoscopic resection; (4) patients with esophageal cancer who underwent radical resection; (5) postoperative ECG monitoring indicated $A F$, which was confirmed by ECG or dynamic ECG records. Exclusion criteria: (1) there was a history of persistent or paroxysmal AF before the operation; (2) the use of antiarrhythmic drugs before the operation; (3) emergency surgery; (4) color
Doppler echocardiography suggested moderate or severe stenosis of the mitral valve; (5) previous mitral valve replacement and mitral valve repair and wedge or segmental resection of lung due to mitral valve disease.

\section{Data collection}

The basic information (age, sex, height, weight, etc.), previous medical history (smoking, chronic lung disease, hypertension, diabetes, heart failure, coronary heart disease, valvular disease, stroke, chronic kidney disease, etc.), medication history, preoperative echocardiography (left anterior and posterior atrium diameter, left ventricular ejection fraction, E/E' at the interventricular septal mitral annulus, etc.) and electrocardiogram (ECG) before and after the operation were recorded. The operation mode, resection scope and histopathology were registered according to the operation record. In addition, we recorded any patients' postoperative death or hospitalization, including hospitalization days, hospitalization costs, etc. According to the CHADS2 score, patients were divided into low-risk (0 points), intermediate-risk (1 point), and high-risk groups ( $\geq 2$ points) [11].

\section{Primary endpoint}

Atrial fibrillation or atrial flutter occurred 30 days after the operation. Atrial fibrillation or atrial flutter lasted at least $5 \mathrm{~min}$ and was recorded by ECG or 24 hour Holter monitor.

\section{Preoperative and postoperative medication}

Hypertensive patients did not stop using antihypertensive drugs during the perioperative period, unless adjustment was needed due to fluctuations in blood pressure. For patients with POAF, drugs such as $\beta$-blockers, amiodarone, digitalis, could be used according to the clinician. All patients entered the anesthesia recovery room after the operation and continued ECG, blood pressure, blood oxygen saturation monitoring, and then transferred to the postoperative monitoring room of thoracic surgery until $72 \mathrm{~h}$ after the operation. The monitoring time could be appropriately delayed according to the condition of the disease.

\section{Statistical analysis}

EpiData 3.0 was used for data entry and SPSS 24.0 was used for statistical analysis. For baseline feature description, normal distribution measurement data were represented as mean \pm standard deviation (SD), non-normal distribution measurement data were represented as M (P25, P75), and count data were represented as frequency (\%). According to the type of data, the $t$ test, Mann-Whit- 
ney $U$ test, $\chi^{2}$ test and Fisher's exact probability method were used for univariate analysis. The variables with statistical $(p<0.1)$ or clinical significance in univariate factor analysis were included in the multifactor logistic regression model, and the independent variables were screened by the step-by-step forward method. The prediction probability was calculated, and the odds ratio (OR) value and $95 \% \mathrm{Cl}$ were analyzed. The Hosmer-Lemeshow goodness-of-fit test was used to evaluate the calibration ability of the prediction model. According to the prediction models of Passman et al., including male sex, preoperative heart rate greater than 72 beats/min, age 55-74 years, and age $\geq 75$ years, the patients included in this study were verified to calculate the prediction probability [12]. The ROC curve was drawn by MEDCAL C 19.0, and the difference between the prediction model of this study and the prediction model reported in the literature was compared. Inspection level $\alpha=0.05$, two-sided inspection $p<0.05$.

\section{Results}

\section{General information}

From January 1, 2017 to December 31, 2018, 3365 patients in thoracic surgery of our hospital received thoracotomy or thoracoscopic resection, radical resection of esophageal cancer. A total of 142 patients with previous AF and AF, 41 patients with antiarrhythmic drugs, 1092 patients who underwent wedge or segmental resection of lung and 18 patients with mitral valve replacement were excluded, and 2072 patients were finally included. There were 124 patients with POAF, and the total incidence of POAF was $5.98 \%$. According to whether there was POAF or not, the patients were divided into the POAF group (124 patients), and non-POAF group (1948 patients). The general information of selected patients is shown in Table I.

\section{Analysis of factors affecting POAF}

The results showed that compared with the non-POAF group, there was a higher percentage of men $(77.4 \%$ vs. $52.9 \%, p<0.001)$, higher BMI $\left((23.3 \pm 2.6) \mathrm{kg} / \mathrm{m}^{2}\right.$ vs. $\left.(22.7 \pm 3.1) \mathrm{kg} / \mathrm{m}^{2}, p=0.031\right)$ and older age (median age: 65 years old vs. 59 years old, $p<0.001$ ) in the POAF group (Table I). The results of echocardiography before the operation showed that the anteroposterior diameter of the left atrium in the POAF group was significantly larger than that in the non-POAF group ((36.3 $\pm 5.6) \mathrm{mm}$ vs. $(32.0 \pm 4.4) \mathrm{mm}, p<0.01)$. There was no significant difference in $E / E$ or left ventricular EF between the two groups $(p>0.05)$. The proportion of esophageal surgery in the AF group was higher than that in the non-AF group (23.0\% vs. $8.2 \%, p<0.001)$. There was no significant differ- ence in CHADS2 score or preoperative and postoperative medication between the two groups $(p>0.05)$. The incidence of postoperative pulmonary infection in the POAF group was significantly higher than that in the non-POAF group $(17.7 \%$ vs. $5.4 \%, p<0.01$ ) (Table I). Among the patients with postoperative pulmonary infection, the incidence of pulmonary infection after esophagectomy was higher than that of pulmonary surgery $(9.1 \%$ vs. $5.4 \%, p<0.01)$.

\section{Postoperative outcome}

The hospitalization days $(9(5,14)$ vs. $11(6.8$, 16.3), $p=0.028$ ) and medical expenses (58594 $(45321,65869)$ vs. 54137 (51681, 69334), $p=$ 0.012 ) in the AF group were significantly higher than those in the non AF group (Table I).

\section{Multifactor logistic regression results}

According to the results of univariate analysis, sex, age, BMI, anteroposterior diameter of the left atrium and surgical organs were included in the logistic regression model. There was no significant correlation between $\mathrm{BMI}$ and POAF. The risk factors of POAF were male sex, age, anteroposterior diameter of left atrium and surgical organs (Table II).

\section{Discrimination and correction}

The AUC was $0.784,95 \% \mathrm{Cl}$ was 0.746 to 0.822 $(p<0.001)$ (Figure 1).

Patients were ranked from low to high incidence of predicted POAF and grouped in deciles to compare the average predicted probability and actual incidence of each group (Figure 2). The Hosmer-Lemeshow test showed that $\chi^{2}=11.9$, $p=0.151(p>0.05)$. The results showed that the model was accurate and the credibility was high.

\section{Discussion}

Lung cancer and esophageal cancer are common cancers in China, and lung cancer ranks the highest in incidence rate and mortality rate of all malignant tumors $[13,14]$. Surgery remains the preferred treatment for lung cancer and esophageal cancer. POAF is a common complication of thoracic surgery, and identifying high-risk groups of POAF in advance is of great clinical significance. There are significant regional differences in the occurrence of POAF. In the European and American population, the incidence is $30-40 \%$, while in the Asian population, it is $15-20 \%$ [15]. The risk factors of POAF include: age, sex, hypertension and other chronic cardiovascular diseases; operation-related factors such as operation type and inflammatory response; and postoperative related factors such as hypoxia, fluid overload and pain 
Table I. General information

\begin{tabular}{|c|c|c|c|}
\hline Parameter & $\begin{array}{l}\text { POAF group } \\
(n=124)\end{array}$ & $\begin{array}{l}\text { Non-POAF group } \\
\quad(n=1948)\end{array}$ & $P$-values \\
\hline Male, $n(\%)$ & $96(77.4)$ & $1030(52.9)$ & $<0.001$ \\
\hline Median age [years] & $65(58,71)$ & $59(51,65)$ & $<0.01$ \\
\hline BMI $\left[\mathrm{kg} / \mathrm{m}^{2}\right]$ & $23.3 \pm 2.6$ & $22.7 \pm 3.1$ & 0.031 \\
\hline \multicolumn{4}{|l|}{ Past medical history } \\
\hline Smoking, $n(\%)$ & $48(38.7)$ & $622(31.9)$ & 0.118 \\
\hline Chronic lung disease, $n(\%)$ & $11(14.9)$ & $97(5.5)$ & 0.64 \\
\hline Hypertension, $n$ (\%) & $21(28.4)$ & $370(21.0)$ & 0.14 \\
\hline Diabetes, $n(\%)$ & $7(9.5)$ & $160(9.1)$ & 0.83 \\
\hline Coronary heart disease, $n$ (\%) & $9(12.2)$ & $189(10.7)$ & 0.69 \\
\hline Heart failure, $n$ (\%) & $3(4.1)$ & $36(2.0)$ & 0.24 \\
\hline Apoplexy, $n(\%)$ & $1(1.4)$ & $16(0.9)$ & 0.5 \\
\hline Preoperative heart rate (beats/min) & $73(66,73)$ & $73(65,73)$ & 0.72 \\
\hline \multicolumn{4}{|l|}{ Preoperative medication, $n$ (\%): } \\
\hline ACEI/ARB & $6(8.1)$ & $138(7.8)$ & 0.82 \\
\hline Beta blocker & $3(4.1)$ & $53(3.0)$ & 0.49 \\
\hline $\mathrm{CCB}$ & $6(8.1)$ & $196(11.1)$ & 0.56 \\
\hline LA [mm] & $36.3 \pm 5.6$ & $32.0 \pm 4.4$ & $<0.01$ \\
\hline LVEF (\%) & $67.1 \pm 5.4$ & $67.8 \pm 5.2$ & 0.25 \\
\hline E/E' & $10.6(8.4,12.4)$ & $9.5(7.8,11.6)$ & 0.009 \\
\hline Operation organs, $n(\%)$ : & & & 0.006 \\
\hline Lung & $88(71.0)$ & $1578(81.0)$ & \\
\hline Esophagus & $36(29.0)$ & $370(19.0)$ & \\
\hline Postoperative pulmonary infection & $22(17.7)$ & $105(5.4)$ & $<0.01$ \\
\hline \multicolumn{4}{|l|}{ Hemoglobin [g/l]: } \\
\hline Before operation & $132.1 \pm 13.7$ & $133.0 \pm 13.2$ & 0.48 \\
\hline After operation & $129.3 \pm 13.4$ & $131.3 \pm 12.2$ & 0.12 \\
\hline CHADS $_{2}$ score, $n(\%):$ & & & 0.37 \\
\hline 0 point & $50(67.6)$ & $1316(74.8)$ & \\
\hline 1 point & $17(23.0)$ & $321(18.2)$ & \\
\hline$\geq 2$ points & $7(9.5)$ & $123(7.1)$ & \\
\hline Hospitalization day & $9(5,14)$ & $11(6.8,16.3)$ & 0.023 \\
\hline Cost (Yuan) & 58594 & 54137 & 0.012 \\
\hline
\end{tabular}

Table II. Multifactor logistic regression results

\begin{tabular}{|lcccccc|}
\hline Items & B & $\begin{array}{c}\text { Standard } \\
\text { error }\end{array}$ & Wald & Freedom & Significance & Exp (B) (95\% Cl) \\
\hline Male & 0.861 & 0.229 & 14.165 & 1 & 0.000 & $2.365(1.511-3.702)$ \\
\hline $\begin{array}{l}\text { Anteroposterior diameter } \\
\text { of left atrium }\end{array}$ & 0.154 & 0.021 & 54.084 & 1 & 0.000 & $1.166(1.120-1.215)$ \\
\hline Operation organ & 0.445 & 0.220 & 4.091 & 1 & 0.043 & $1.560(1.014-2.401)$ \\
\hline Age & 0.053 & 0.012 & 21.132 & 1 & 0.000 & $1.055(1.031-1.079)$ \\
\hline Constant & -11.946 & 0.955 & 156.568 & 1 & 0.000 & 0.000 \\
\hline
\end{tabular}




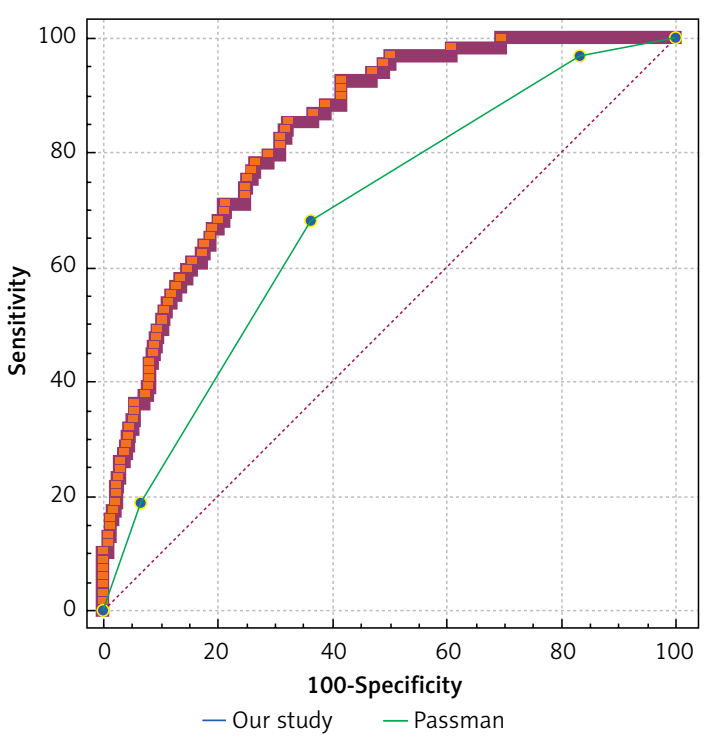

Figure 1. Comparison of ROC curve between current research prediction model and Passman prediction model

stimulation [8]. Previous studies have shown that the risk factors of POAF include race, old age, male sex, preoperative NT proBNP increase, left atrial increase, previous heart failure history, preoperative chemotherapy, tumor stage, operation type, etc. [16-19]. However, the results are not consistent $[1,8]$. Based on the relevant risk factors, several predictive models have been established, such as $\mathrm{CHADS}_{2}, \mathrm{CHA}_{2} \mathrm{DS}_{2}$-VASc, HACTH and POAF [12, 20-22], to identify patients with high risk of POAF before the operation, and have been verified by relevant clinical studies [22]. However, the above prediction models are mostly based on the research data of patients with cardiac surgery in Europe and America, and the results are not necessarily suitable for patients with non-cardiac surgery.

The prediction model of POAF for patients with non-cardiac surgery is not mature, and the published results are based on the European and American population $[11,12,18,19]$. The related risk factors include age, sex, high $\mathrm{BMI}$, high preoperative BNP, history of atrial fibrillation, preoperative heart rate higher than 72 beats/min, blood transfusion and surgical resection range. The risk factors used in different prediction models are not the same, but the risk factors in all prediction models include age and male sex. In this study, 2072 patients undergoing non-cardiac surgery were analyzed retrospectively. After excluding the confounding factors, we found that age, sex, anteroposterior diameter of left atrium and surgical organs were the risk factors of POAF in the local patients. On this basis, we established a predictive model of POAF, and the results showed that the model had high diagnostic value, sensitivity and specificity.

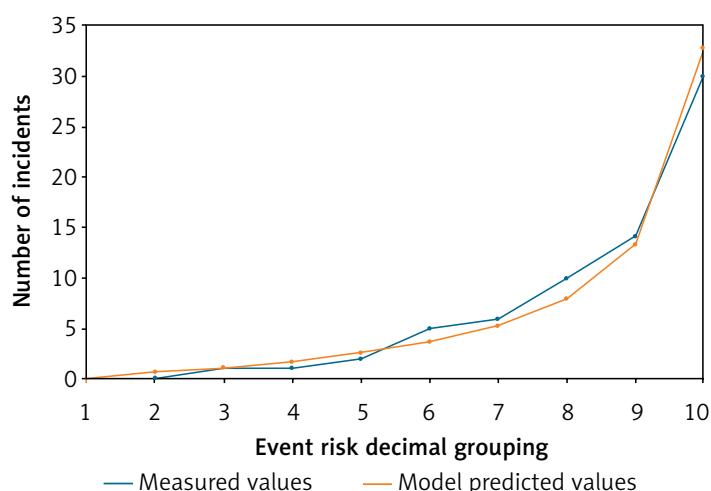

Figure 2. Schematic diagram of comparison between measured values and model predicted values of POAF

Consistent with previous studies, in this study, age and sex are also risk factors for POAF, and elderly or male patients are at higher risk of POAF. In addition, the operation organ is a predictor of POAF. Among them, the risk of POAF after esophagectomy was significantly higher than that of pulmonary tumor resection. Most previous studies have confirmed that the operation type or surgical resection range is related to the occurrence of POAF. Generally speaking, the larger the resection range, the higher the risk of POAF. However, a small number of studies do not support this conclusion. It has been reported that the probability of POAF is $2-4 \%, 10-15 \%$ and $20-30 \%$ after segmental resection, lobectomy and pneumonectomy, respectively [23]. A study involving 2588 patients undergoing thoracic non-cardiac surgery showed that, compared with wedge resection, segmental resection did not increase the incidence of POAF, while pneumonectomy, lobectomy and esophagectomy significantly increased the incidence of POAF, with a relative risk of 8.91 , 3.89 and 2.95, respectively [18]. Onaitis mark et al. also confirmed that the expansion of surgical resection range was related to the increased incidence of POAF [24]. In David Amar's prediction model of POAF, surgical resection range is also a predictor [19], but in Passman's study, surgical resection range is not a risk predictor of POAF [12].

The results of this study showed that the left atrium anteroposterior diameter in AF group was significantly higher than that in the non-AF group. Multivariate logistic regression showed that left atrium diameter greater than $35 \mathrm{~mm}$ was a strong predictor of POAF. Several previous studies have shown that abnormalities of left atrium structure and function are risk factors for $\operatorname{POAF}[17,25,26]$. However, there is a view that the determination of the relevant parameters of the left atrium requires a professional examiner to test, which will increase the medical cost [19]. Furthermore, elevated BNP or NT-proBNP is an effective predic- 
tor of POAF $[16,19,27]$. However, recent studies have shown that the predictive value of the left atrial diastolic volume index for POAF is greater than that of BNP [25]. Unlike previous studies, our study is the first to incorporate them into the POAF prediction model. In China, the cost of echocardiographic examination is almost the same as that of BNP or NT-proBNP, and echocardiography can provide a lot of structural and functional assessment of the heart, which is very important for preoperative assessment of lung and esophagus-related surgery. Therefore, patients in this study underwent routine echocardiography before surgery, but did not undergo a BNP or NT-proBNP test. It is easy to measure the left atrium anteroposterior diameter by echocardiography with good repeatability. Anile et al. found that the area of the left atrium measured by preoperative echocardiography (more than $20 \mathrm{~mm}^{2}$ ) has predictive value for the occurrence of POAF in patients with lobectomy and pneumonectomy [17]. However, $\mathrm{Ai}$ et al. concluded that left atrial volume index and left ventricular diastolic function such as E/E' ratio had no predictive value for the occurrence of AF after surgery in patients with lung cancer. The results of this study showed that the incidence of pulmonary infection in the POAF group was higher than that in the non-POAF group. Pulmonary infection may increase the incidence of atrial fibrillation, but its causal relationship cannot be accurately determined in this study.

The results of this study showed that CHADS2 score has no predictive value for the occurrence of POAF in the population included in this study. ROC analysis showed that the predictive ability of the model constructed in this study was significantly higher than that of the Passman and Svetlana models [11, 12]. David Amar et al. included BMI in their prediction model of POAF. In this study, there were not similar results. The reason may be due to the large difference in body size between Chinese and European and American people. In conclusion, our study showed that the prediction model of POAF based on European and American populations was not suitable for the Chinese population, and people in different regions may need different prediction tools. Increasing left atrial diameter on the basis of age can significantly improve the diagnostic value of the predictive model.

Moreover, in this study, the incidence of POAF was $5.98 \%$. The incidence of POAF was significantly lower than that of large-scale clinical case studies in Europe and America [11, 12, 18, 24, 28-31], which was close to the clinical studies of Xie and Mayo [32-34]. The reason may be that the subjects were younger than in similar studies, and the proportion of chronic diseases such as hypertension was lower.
This study also has some limitations. First, this is a retrospective study. Second, it is a single center study. Third, the ECG monitoring time in this study was about $72 \mathrm{~h}$, which may omit part of atrial fibrillation after $72 \mathrm{~h}$, especially silent AF.

In conclusion, through the retrospective analysis of data of 2072 patients undergoing resection of lung and esophageal tumors, we found that age, sex, left atrium anteroposterior diameter and operation type were the high risk factors of POAF in Chinese patients after extrathoracic non-cardiac surgery, and on this basis, we established a prediction model. The model has good sensitivity and specificity, and is suitable for the prediction of POAF in Chinese patients undergoing extrathoracic surgery.

\section{Conflict of interest}

The authors declare no conflict of interest.

\section{References}

1. Fernando HC, Jaklitsch MT, Walsh GL, et al. The Society of Thoracic Surgeons practice guideline on the prophylaxis and management of atrial fibrillation associated with general thoracic surgery: executive summary. Ann Thorac Surg 2011; 92: 1144-52.

2. Gialdini G, Nearing K, Bhave PD, et al. Perioperative atrial fibrillation and the long-term risk of ischemic stroke. JAMA 2014; 312: 616-22.

3. Bhave PD, Goldman LE, Vittinghoff E, Maselli J, Auerbach A. Incidence, predictors, and outcomes associated with postoperative atrial fibrillation after major noncardiac surgery. Am Heart J 2012; 164: 918-24.

4. Frendl G, Sodickson AC, Chung MK, et al. 2014 AATS guidelines for the prevention and management of perioperative atrial fibrillation and flutter for thoracic surgical procedures. J Thorac Cardiovasc Surg 2014; 148: e153-93.

5. Maesen B, Nijs J, Maessen J, Allessie M, Schotten U. Post-operative atrial fibrillation: a maze of mechanisms. Europace 2012; 14: 159-74.

6. Butt JH, Olesen JB, Havers-Borgersen E, et al. Risk of thromboembolism associated with atrial fibrillation following noncardiac surgery. J Am Coll Cardiol 2018; 72: 2027-36.

7. Banach M, Mariscalco G, Ugurlucan M, Mikhailidis DP, Barylski M, Rysz J. The significance of preoperative atrial fibrillation in patients undergoing cardiac surgery: preoperative atrial fibrillation--still underestimated opponent. Europace 2008; 10: 1266-70.

8. Vallurupalli S, Shanbhag A, Mehta JL. Controversies in postoperative atrial fibrillation after noncardiothoracic surgery: clinical and research implications. Clin Cardiol 2017; 40: 329-32.

9. Banach M, Kourliouros A, Reinhart KM, et al. Postoperative atrial fibrillation - what do we really know? Curr Vasc Pharmacol 2010; 8: 553-72.

10. Mariscalco G, Biancari F, Zanobini M, et al. Bedside tool for predicting the risk of postoperative atrial fibrillation after cardiac surgery: the POAF score. J Am Heart Assoc 2014; 3: e000752.

11. Kotova S, Wang M, Lothrop K, Grunkemeier G, Merry HE, Handy JR. CHADS score predicts postoperative atrial 
fibrillation in patients undergoing elective pulmonary lobectomy. Ann Thorac Surg 2017; 103: 1566-72.

12. Passman RS, Gingold DS, Amar D, et al. Prediction rule for atrial fibrillation after major noncardiac thoracic surgery. Ann Thorac Surg 2005; 79: 1698-703.

13. Zhou M, Wang H, Zeng X, et al. Mortality, morbidity, and risk factors in China and its provinces, 1990-2017: a systematic analysis for the Global Burden of Disease Study 2017. Lancet 2019; 394: 1145-58.

14. Rongshou Z, Kexin S, Siwei Z, et al. Analysis of cancer prevalence in China in 2015. Chinese J Cancer 2019; 41: 19-28.

15. Vlachos K, Letsas KP, Korantzopoulos P, et al. Prediction of atrial fibrillation development and progression: current perspectives. World J Cardiol 2016; 8: 267-76.

16. Cardinale D, Colombo A, Sandri MT, et al. Increased perioperative $\mathrm{N}$-terminal pro-B-type natriuretic peptide levels predict atrial fibrillation after thoracic surgery for lung cancer. Circulation 2007; 115: 1339-44.

17. Anile M, Telha V, Diso D, et al. Left atrial size predicts the onset of atrial fibrillation after major pulmonary resections. Eur J Cardiothorac Surg 2012; 41: 1094-7.

18. Vaporciyan AA, Correa AM, Rice DC, et al. Risk factors associated with atrial fibrillation after noncardiac thoracic surgery: analysis of 2588 patients. J Thorac Cardiovasc Surg 2004; 127: 779-86.

19. Amar D, Zhang H, Tan KS, Piening D, Rusch VW, Jones DR. A brain natriuretic peptide-based prediction model for atrial fibrillation after thoracic surgery: development and internal validation. J Thorac Cardiovasc Surg 2019; 157: 2493-9.e1.

20. de Vos CB, Pisters R, Nieuwlaat R, et al. Progression from paroxysmal to persistent atrial fibrillation clinical correlates and prognosis. J Am Coll Cardiol 2010; 55: 725-31.

21. Mariscalco G, Biancari F, Zanobini M, et al. Bedside tool for predicting the risk of postoperative atrial fibrillation after cardiac surgery: the POAF score. J Am Heart Assoc 2014; 3: e000752.

22. Burgos LM, Seoane L, Parodi JB, et al. Postoperative atrial fibrillation is associated with higher scores on predictive indices. J Thorac Cardiovasc Surg 2019; 157: 2279-86.

23. Shrivastava V, Nyawo B, Dunning J, Morritt G. Is there a role for prophylaxis against atrial fibrillation for patients undergoing lung surgery? Interact Cardiovasc Thorac Surg 2004; 3: 656-62.

24. Onaitis M, D'Amico T, Zhao Y, O'Brien S, Harpole D. Risk factors for atrial fibrillation after lung cancer surgery: analysis of the Society of Thoracic Surgeons general thoracic surgery database. Ann Thorac Surg 2010; 90: 368-74.

25. Brecher O, Gulati H, Roistacher N, et al. Preoperative echocardiographic indices of diastolic dysfunction and brain natriuretic peptide in predicting postoperative atrial fibrillation after noncardiac surgery. Anesthesia Analgesia 2017; 124: 1099-104.

26. Raman T, Roistacher N, Liu J, et al. Preoperative left atrial dysfunction and risk of postoperative atrial fibrillation complicating thoracic surgery. J Thorac Cardiovasc Surg 2012; 143: 482-7.

27. Toufektzian L, Zisis C, Balaka C, Roussakis A. Effectiveness of brain natriuretic peptide in predicting postoperative atrial fibrillation in patients undergoing non-cardiac thoracic surgery. Interact Cardiovasc Thorac Surg 2015; 20: 654-7.

28. Ai D, Lasala J, Mehran JR, Xu G, Banchs J, Cata JP. Preoperative echocardiographic parameters of diastolic dys- function did not provide a predictive value for postoperative atrial fibrillation in lung and esophageal cancer surgery. J Cardiothor Vasc Anesth 2015; 29: 1127-30.

29. Amar D, Burt ME, Bains MS, Leung DH. Symptomatic tachydysrhythmias after esophagectomy: incidence and outcome measures. Ann Thorac Surg 1996; 61: 1506-9.

30. Mariscalco G, Klersy C, Zanobini M, et al. Atrial fibrillation after isolated coronary surgery affects late survival. Circulation 2008; 118: 1612-8.

31. Banach M, Rysz J, Drozdz JA, et al. Risk factors of atrial fibrillation following coronary artery bypass grafting: a preliminary report. Circ J 2006; 70: 438-41.

32. Allen MS, Deschamps C, Jones DM, Trastek VF, Pairolero PC. Video-assisted thoracic surgical procedures: the Mayo experience. Mayo Clinic Proc 1996; 71: 351-9.

33. Daniels LJ, Balderson SS, Onaitis MW, D'Amico TA. Thoracoscopic lobectomy: a safe and effective strategy for patients with stage I lung cancer. Ann Thorac Surg 2002; 74: 860-4.

34. Xie K, Zhang W, Fang J, et al. Prevalence and risk factors of atrial fibrillation during lung and esophageal surgery: a prospective observational study. Medicine 2018; 97 : e11549. 